University of Nebraska - Lincoln

DigitalCommons@University of Nebraska - Lincoln

\title{
$5-2011$
}

\section{First Microsatellites From Spodoptera frugiperda (Lepidoptera: Noctuidae) and Their Potential Use for Population Genetics}

\author{
R. S. Arias \\ USDA-ARS MSA Genomics and Bioinformatics Research Unit, renee.arias@usda.gov \\ Carlos A. Blanco \\ USDA-ARS Southern Insect Management Research Unit, carlos.a.blanco@aphis.usda.gov \\ Maribel Portilla \\ USDA-ARS Southern Insect Management Research Unit \\ Gordon L. Snodgrass \\ USDA-ARS Southern Insect Management Research Unit \\ Brian E. Scheffler \\ USDA-ARS, brian.scheffler@ars.usda.gov
}

Follow this and additional works at: https://digitalcommons.unl.edu/usdaarsfacpub

Part of the Agricultural Science Commons

Arias, R. S.; Blanco, Carlos A.; Portilla, Maribel; Snodgrass, Gordon L.; and Scheffler, Brian E., "First Microsatellites From Spodoptera frugiperda (Lepidoptera: Noctuidae) and Their Potential Use for Population Genetics" (2011). Publications from USDA-ARS / UNL Faculty. 890.

https://digitalcommons.unl.edu/usdaarsfacpub/890

This Article is brought to you for free and open access by the U.S. Department of Agriculture: Agricultural Research Service, Lincoln, Nebraska at DigitalCommons@University of Nebraska - Lincoln. It has been accepted for inclusion in Publications from USDA-ARS / UNL Faculty by an authorized administrator of DigitalCommons@University of Nebraska - Lincoln. 


\title{
First Microsatellites From Spodoptera frugiperda (Lepidoptera: Noctuidae) and Their Potential Use for Population Genetics
}

\author{
RENÉE S. ARIAS, ${ }^{1,2}$ CARLOS A. BLANCO,${ }^{3,4}$ MARIBEL PORTILLA, ${ }^{3}$ GORDON L. SNODGRASS, ${ }^{3}$ \\ AND BRIAN E. SCHEFFLER ${ }^{1}$
}

Ann. Entomol. Soc. Am. 104(3): 576-587 (2011); DOI: 10.1603/AN10135

\begin{abstract}
This is the first report of sequence-specific microsatellite markers (simple sequence repeats [SSRs] ) of fall armyworm, Spodoptera frugiperda (J.E. Smith) (Lepidoptera: Noctuidae), an economically important pest of crops on the Americas. We isolated 192 microsatellite markers by using pyrosequencing and screened 15 individuals from eight isofamilies collected from three geographical areas: Puerto Rico (PR), Texas (TX), and Mississippi (MS). Isofamilies resistant to Cry toxins from Bacillus thuringiensis (Berliner) (Bacillales: Bacillaceae) also were included. Cluster analysis was performed to determine the potential use of these SSRs in discriminating populations, and colonies were grouped with a reliability of $100 \%$ estimated by bootstrap. In this analysis, colonies from TX grouped away from those from PR, but the two MS isofamilies grouped with TX and PR separately. Genetic distance within isofamilies ranged between 0.22 and 0.56 , and the minimum distance between isofamilies was 0.83 . Unique pattern informative combination (UPIC) scores were calculated, and the 80 SSR markers that had UPIC scores of $\geq 1$ are listed according to their discriminating potential. UPIC scores allow reducing costs by choosing fewer and highly informative markers for future studies. From the best 125 markers, 103 had a maximum of two alleles per sample, making them ideal candidates for population genetic studies. BLAST screening of the sequences points to potential biological meaning of marker polymorphisms. The percentage of alleles shared by the three geographic areas was $14 \%$. The markers reported will significantly enrich the pool of molecular markers available for S. frugiperda. In addition, they could be used for monitoring migration of populations, in the development of biocontrol agents and for management practices in general.
\end{abstract}

RESUMEN Esta es la primera publicación de microsatelites de Spodoptera frugiperda (J.E. Smith) (Lepidoptera: Noctuidae), una plaga importante del continente Americano. Hemos aislado 192 marcadores de microsatelites usando un pyrosequenciador, y analizamos 15 individuos de eight isofamilias colectadas de tres áreas geográficas, Puerto Rico (PR), Texas (TX) y Mississippi (MS), incluyendo isofamilias resistentes y susceptibles a Bacillus thuringiensis (Berliner) (Bacillales: Bacillaceae). Análisis de cluster SE realizó con el propósito de determinar el potencial discriminatorio de los microsatélites. Este agrupo las isofamilias de TX distantes de las isofamilias de PR, mientras que las de MS SE agruparon con TX y con PR separadamente. La distancia genética dentro de isofamilias fue de 0.22 a 0.56 , mientras que la distancia mínima entre isofamilias fue 0.83 . Un total de 80 marcadores que tuvieron valores de UPIC $\geq 1$ como potencial discriminante son presentados. Valores de UPIC permiten reducir costos y elegir marcadores que brindan la máxima variabilidad genética en estudios posteriores. Los marcadores listados pueden contribuir significativamente al número de marcadores moleculares disponibles para $S$. frugiperda. De los mejores 125 markers, 103 presentaron un máximo de two alelos por muestra, lo que los hace buenos candidatos para estudios de genética poblacional. Resultados de BLAST indicarían potencial significado biológico del polimorfismo. El porcentaje de alelos compartidos por las tres regiones

Mention of trade names or commercial products in this article is solely for the purpose of providing specific information and does not imply recommendation or endorsement by the U.S. Department of Agriculture.

${ }^{1}$ USDA-ARS MSA Genomics and Bioinformatics Research Unit, 141 Experiment Station Rd., Stoneville, MS 38776.

2 Corresponding author: e-mail, carlos.a.blanco@aphis.usda.gov.

${ }^{3}$ USDA-ARS Southern Insect Management Research Unit, 141 Experiment Station Rd., Stoneville, MS 38776.

${ }^{4}$ USDA-APHIS Biotechnology Regulatory Services, 4700 River Rd., Riverdale, MD 20737.

This article is a U.S. government work, and is not subject to copyright in the United States. 
geográficas fue $14 \%$. Además, estos marcadores podrían ser usados para monitorear migración de poblaciones, en el desarrollo de agentes de control biológico, en programas de mejoramiento, y para prácticas de manejo en general.

KEY WORDS pyrosequencing, fall armyworm, simple sequence repeat, molecular markers, fingerprinting

The fall armyworm, Spodoptera frugiperda (J.E. Smith) (Lepidoptera: Noctuidae), is endemic to the American continent (Johnson 1987) where it causes economic losses in important crops such as maize (Zea mays L.), cotton (Gossipium spp. L.), alfalfa (Medicago sativa L.), rice (Oryza sativa L.), and grasses (Sparks 1986). In a crop as important as maize, which has a world production of $\approx 727,000 \mathrm{MT}$ (Index-Mundi 2009), losses caused by S. frugiperda can reach up to 20\% (Marenco et al. 1992, Polanczyk et al. 2000), and if this insect is not controlled, it can reduce maize yields by up to $73 \%$ (Hruska and Gladstone 1988). The control of $S$. frugiperda mostly relies on timely applications of insecticide aimed at susceptible larval developmental stages during incipient plant damage, which gives to this control tactic a narrow window of opportunity for optimal treatment. In maize alone, the amount of insecticide used can reach 30,000 tons of active ingredient per year (Blanco et al. 2010). The alternative method of control is the use of transgenic crops expressing Cry toxins from Bacillus thuringiensis (Berliner) (Bt; Bacillales: Bacillaceae) (Jenkins 1999). However, S. frugiperda has shown some level of tolerance to Cryl $\mathrm{F}$ toxin (Adamczyk et al. 1997, Chilcutt et al. 2007, de Souza et al. 2009). In particular, Puerto Rico (PR) fall armyworm populations have already shown high tolerance to the $\delta$-endotoxin Cryl $\mathrm{F}$ of Bt (Tabashnik et al. 2009, Nagoshi et al. 2010). Because Puerto Rico is only $1,500 \mathrm{~km}$ away from the nearest U.S. coastline, and this island can be affected by numerous yearly weather systems that commonly move northward during high activity of fall armyworm moths, it creates the possibility that this insect, putatively resistant to $\mathrm{Bt}$, can easily move to the continental United States. In fact, Young (1979) and Westbrook and Sparks (1986) have documented the passive movement of this insect via weather atmospheric transport. Thus, numerous questions have arisen regarding the migration of populations, possible interbreeding, and development of resistance in view of the increasing use of transgenic crops expressing Bt toxins.

To control this pest and develop effective management practices it is important to understand the migratory patterns, genetic diversity of the existing populations, and potential development of new populations. So far, two strains have been described of $S$. frugiperda according to their host preference and behavior, but these strains present no morphological differences (Pashley 1986, Pashley et al. 1985, Nagoshi and Meagher 2009) and are only distinguished by molecular methods (Pashley et al. 1985, Nagoshi and Meagher 2003, Martinelli et al. 2006, Machado et al. 2008, Velez-Arango et al. 2008).
Numerous studies have been performed to understand the migrations of S. frugiperda in the United States, with sometimes inconsistent results regarding annual migrations, as pointed out by Nagoshi and Meagher (2008). Some molecular tools have been developed to monitor migrations and/or crosses of this insect. For example, two polymorphic loci in the sequence analysis of the mitochondrial cytochrome oxidase subunit I gene (COI) can distinguish four haplotypes in S. frugiperda populations across America (Pashley 1989), and the proportions of these haplotypes have been used to identify populations (Nagoshi 2010). Other molecular tools developed to assist in the identification of populations of S. frugiperda are a short-repeat element known as Found in Rice (FR) that maps to sex chromosomes (Lu et al. 1994, Nagoshi and Meagher 2003), and polymorphisms in the DNA sequence of the triose phosphate isomerase gene (Tpi) (Nagoshi 2010). However, the need for a multilocus approach to understand the biology of $S$. frugiperda has been emphasized by Prowell et al. (2004), who used combination of molecular tools, including allozymes, amplified fragment length polymorphisms (AFLPs), and mitochondrial DNA to study speciation and introgression in S. frugiperda. In fact, the use of large number of loci is more likely to contain information about gene flow during older events even if analyzing small samples (Wang and Hey 2010).

Microsatellites, also known as simple sequence repeats (SSRs), have been the most widely applied class of molecular markers used in genetic studies with applications in many fields of genetics, including genetic conservation, population genetics, molecular breeding, and paternity testing (Ellegren 2004). This range of applications is possible because microsatellite markers are codominant, multiallelic, and highly reproducible; have high resolution; and are based on polymerase chain reaction (PCR) (Oliveira et al. 2006). As a convention, SSRs are regions in the genome where a group of bases $(1-8 \mathrm{bp})$ are repeated in tandem (Richard et al. 2008). These regions can be isolated either by data mining of existing sequences or by generating SSR-enriched libraries (Kijas et al. 1994, Zane et al. 2002).

Genetic resources of S. frugiperda are significant, i.e., there are 1,110 nucleotide entries in National Center for Biotechnology Information (NCBI; eight correspond to midgut) and 65,403 expressed sequence tags (ESTs; 11,192 correspond to midgut) reported. However, there have been no publications on microsatellite markers developed for S. frugiperda. There have been only eight microsatellite markers developed for a related species, Spodoptera exempta (Walker), where it is mentioned that two of the mark- 
Table 1. Origin of $S$. frugiperda colonies including their corresponding hosts and locations in the United States

\begin{tabular}{ccccc}
\hline $\begin{array}{c}\text { Colony } \\
\text { name }\end{array}$ & $\begin{array}{c}\text { Geographic } \\
\text { origin }^{a}\end{array}$ & Host & $\begin{array}{c}\text { Bt } \\
\text { resistance }\end{array}$ & Generation \\
\hline 456 & PR & Maize & Yes & F3 \\
512 & PR & Maize & Yes & F3 \\
751 & PR & Maize & Yes & F3 \\
778 & MS & Grass & No & F2 \\
957 & MS & Maize & No & F2 \\
980 & TX & Maize & No & F2 \\
985 & TX & Maize & No & F2 \\
989 & TX & Maize & No & F2 \\
\hline
\end{tabular}

The letters A and B used throughout the manuscript and cluster analysis correspond to DNA samples extracted from two individuals from each population.

${ }^{a}$ PR, Puerto Rico; TX, Texas; MS, Mississippi.

ers also amplified S. frugiperda (Ibrahim et al. 2004), and one microsatellite from rice that also amplified a DNA sample of $S$. frugiperda (Zhao and Kochert 1993). Thus, in the current study we used an effective method of microsatellite isolation (Techen et al. 2010), developed sequence-specific microsatellite libraries of $S$. frugiperda, and tested the markers on eight isofamilies from three geographic locations ( $\mathrm{Ta}-$ ble 1). Using cluster analysis and unique pattern informative combination (UPIC) software, we determined the most informative markers and their potential usefulness for multilocus population studies of S. frugiperda. In addition, BLASTx and BLASTn screening of the sequences point to potential biological meaning of the marker polymorphisms and could be the basis for future studies.

\section{Materials and Methods}

Insect Collection and Rearing. Field-collected S. frugiperda larvae (Passoa 1991) were reared in insect artificial diet (Blanco et al. 2009) until adults were obtained. Single pairs from each geographic location formed isofamilies, and their second and third generation $\left(\mathrm{F}_{2} / \mathrm{F}_{3}\right)$ were tested on serial dilutions of $\mathrm{Bt}$ purified proteins (Blanco et al. 2010; Table 1). All isofamilies were derived from maize sampled in College Station, TX (hereafter TX); Santa Isabel, Puerto Rico (PR), and Stoneville, MS (MS), except isofamily 778 that was collected from grasses in MS.

Isolation of Microsatellites. DNA was extracted from S. frugiperda colony 957 , from the $\mathrm{F}_{2}$ generation (Table 1). Only thorax tissue was used for this extraction to avoid contamination with the gut microflora. DNA was extracted with DNeasy Plant Maxi kit (QIAGEN, Valencia, CA), and SSR-enriched libraries were generated following the protocol of Techen et al. (2010), briefly described here. DNA was digested with restriction enzymes AluI, HaeII, DraI, RsaI, and HpyCH4IV (New England Biolabs, Ipswich, MA) individually and with combinations of RsaI+HaeIII and DraI+AluI. The blunt-end DNA fragments were A-tailed with Taq-DNA Polymerase (Promega, Madison, WI) in the presence of dATP for $2 \mathrm{~h}$ and then ligated for $3 \mathrm{~h}$ at $16^{\circ} \mathrm{C}$ to a linker made from oligonucleotides (oligos) SSRLIBF3, 5'-
CGGGAGAGCAAGGAAGGAGT-3' ${ }^{\prime}$ and SSRLIBR3, 5'Phos-CTCCTTCCTTGCTCTCTCCCGAAAA-3' (Techen et al. 2010). The ligated fragments were purified with MinElute (QIAGEN) and amplified by 20 cycles of PCR by using primer SSRLIBF3 and High Fidelity DNA Polymerase (Invitrogen, Carlsbad, CA) at $94^{\circ} \mathrm{C}$ for $30 \mathrm{~s}$, $60^{\circ} \mathrm{C}$ for $30 \mathrm{~s}$, and $68^{\circ} \mathrm{C}$ for $90 \mathrm{~s}$. The amplified products were hybridized to three groups of biotinylated oligo repeats, similar to the groups listed by Glenn and Schable (2005): group $2\left[(\mathrm{AG})_{12},(\mathrm{AAC})_{6},(\mathrm{AAG})_{8},(\mathrm{ACT})_{12}\right.$, $\left.(\mathrm{ATC})_{8}\right]$, group $3\left[(\mathrm{AAAC})_{6},(\mathrm{AAAG})_{6},(\mathrm{AATC})_{6}\right.$, $\left.(\mathrm{AATG})_{6},(\mathrm{ACAG})_{6},(\mathrm{ACCT})_{6},(\mathrm{ACTC})_{6},(\mathrm{ACTG})_{6}\right]$, and group $4\left[(\mathrm{AAAT})_{8},(\mathrm{AACT})_{8},(\mathrm{AAGT})_{8},(\mathrm{ACAT})_{8}\right.$, $\left.(\mathrm{AGAT})_{8}\right]$, primers were purchased from Invitrogen. The final concentration of each oligo in the mix was 1 $\mu \mathrm{M}$, and $2 \mu \mathrm{l}$ of each oligo mix was used in $50-\mu \mathrm{l}$ hybridization reactions. Hybridizations were performed in a gradient thermocycler at $95^{\circ} \mathrm{C}$ for $10 \mathrm{~min}$, followed by $3 \mathrm{~h}$ at the annealing temperature using a gradient block at $50^{\circ} \mathrm{C}$ for group 2 and 4 and $53^{\circ} \mathrm{C}$ for group 3 , followed by an extension step of $10 \mathrm{~min}$ at $68^{\circ} \mathrm{C}$ in the presence of High Fidelity Taq Polymerase (Invitrogen) as indicated in Hayden et al. (2002). Sequences containing repeats were captured using streptavidin-coated magnetic beads M-270 (Invitrogen, Carlsbad, CA) in a Labquake tube shaker/rotator (Barnstead/Thermoline, Dubuque, IA) at $22^{\circ} \mathrm{C}$ for $1 \mathrm{~h}$ (Kijas et al. 1994). After binding, the beads were washed with $0.5 \times$ standard saline citrate $(\mathrm{SSC})$ at ambient temperature and $0.5 \times \mathrm{SSC}$ at $50^{\circ} \mathrm{C}$ for $5 \mathrm{~min}$ each. Elution of the DNA from the biotinylated oligos was done with $60 \mu \mathrm{l}$ of MilliQ water (Millipore, Billerica, $\mathrm{MA}$ ) at $96^{\circ} \mathrm{C}$ for $5 \mathrm{~min}$, twice. The eluate was PCR amplified for 10 cycles as indicated for the ligation step. PCR products were sequenced by pyrosequencing in 1/16th of a plate 454-GS FLX (Roche Diagnostics, Indianapolis, IN) by using a GS Titanium Sequencing kit XLR70 (70 by 75 Titanium Pico-Titer Plates, Roche, Branford, CT; 200 cycles, ), using 1/16th of a plate. Sequences were assembled with 454 gsAssembler version 2.0 (Roche). Repeats were searched using SSRFinder (Sharopova et al. 2002), and primers were designed using Primer3 (Rozen and Skaletsky 2000) with stringent parameter conditions: annealing temperature $(\mathrm{Tm}), 63^{\circ} \mathrm{C}$ optimum; $\left(60 / 65^{\circ} \mathrm{C}\right)$, min/max; length, 24 -bp optimum; (20/28 bp), $\mathrm{min} / \mathrm{max}$; 3'GC clamp; and maximum overlap of repeat within the primer, $5 \mathrm{bp}$. To simplify the recording of the repeated motifs, circular permutations, and reverse complements of the motif sequences were grouped together as one type, i.e., AAC, ACA, CAA, GTT, TGT, TTG were recorded as AAC. Any library/ sequence information requirements can be addressed to B.E.S. at brian.scheffler@ars.usda.gov.

Fingerprinting and Cluster Analysis. Using stringent conditions in Primer3 software, we designed 215 primers on the flanking regions of the repeats and tested 192 of them on 15 individuals from eight $S$. frugiperda colonies from three geographical areas as detailed in Table 1. Fourth and fifth instars of S. frugiperda were used. From colony 751, the DNA extracted from one individual was sufficient to run 96 markers but not enough for all 192 markers used in this study. These isofamilies are maintained by Dr. M. 
Portilla (Southern Insect Management Research Unit, Stoneville, MS). Forward SSR primers were $5^{\prime}$ tailed with the sequence 5'-CAGTTTTCCCAGTCACGAC-3' to permit product labeling, and reverse primers were tailed at the $5^{\prime}$ end with the sequence $5^{\prime}$ GTTT-3' to promote nontemplate adenylation (Brownstein et al. 1996). Primer 5'-CAGTTTTCCCAGTCACGAC-3' labeled with 6-carboxy-fluorescein (FAM) (Integrated DNA Technologies, Inc., Coralville, IA) was used for amplification of 10-ng DNA by using Titanium TaqDNA Polymerase (Clontech, Mountain View, CA) in 5- $\mu$ l reactions on an M\&J thermal cycler (Bio-Rad Laboratories, Hercules, CA) at $95^{\circ} \mathrm{C}$ for $1 \mathrm{~min}, 60^{\circ} \mathrm{C}$ for $1 \mathrm{~min}$ (two cycles), $95^{\circ} \mathrm{C}$ for $30 \mathrm{~s}, 60^{\circ} \mathrm{C}$ for $30 \mathrm{~s}, 68^{\circ} \mathrm{C}$ for $30 \mathrm{~s}(27$ cycles), and a final extension at $68^{\circ} \mathrm{C}$ for $4 \mathrm{~min}$. Fluorescently labeled PCR fragments were analyzed on an ABI 3730XL DNA Analyzer, and data were processed using GeneMapper version 3.7 (both from Applied Biosystems, Foster City, CA). Presence of alleles was converted to a binary matrix. Cluster analysis of SSR marker results for $S$. frugiperda isolates was performed using the unweighted paired group method and arithmetic averages (unweighted pair-group method with arithmetic average) with the Nei's coefficient, algorithm implemented in the SAHN program of NTSYSpc version 2.2 (Exeter Software, Setauket, NY). The confidence levels for the dendrograms were assessed by bootstrap resampling (5,000 replicates) (Felsenstein 1985, Efron et al. 1996) by using WINBOOT (available on line from International Rice Research Institute, Metro Manila, Philippines).

Percentage of Multiallelic Loci, UPICs, BLAST, Number of Effective Alleles, and Expected and Observed Heterozygosity. The percentage of multiallelic loci was calculated for each individual across the 120 SSR markers used for cluster analysis. We also calculated UPIC to identify the most informative markers and marker combinations that could discriminate all the colonies screened. The percentage of multiallelic loci (ML) and UPIC scores were both calculated using UPIC Perl scripts (Arias et al. 2009). The DNA sequences used in this study were screened using BLASTx and BLASTn (Altschul et al. 1990) in NCBI databases, and those with significant hits were indicated on each marker. Effective alleles and heterozygosity were calculated for each locus (Weir 1990).

\section{Results}

DNA Isolation, Repeat Symmetry, and Frequency. In total, 4,806 reads were assembled in 1,904 contigs where 474 SSRs were detected. The average length of the contigs containing SSRs was $337 \mathrm{bp}$ with an average of eight reads per contig. The most abundant repeat motif in these libraries was $\mathrm{AC}$, followed by ATC, AG, and AAG. A summary of the abundance of each repeat motif is shown in Table 2 . We submitted $185 \mathrm{~S}$. frugiperda sequences in total containing microsatellites to GenBank (NCBI), with accession numbers HM752580-HM752766, the numbers indicated in marker ID in Table 3, correspond to the contig num-
Table 2. Motifs and motif frequencies detected on microsatellite-enriched libraries of $S$. frugiperda

\begin{tabular}{lclc}
\hline \hline Motif & Frequency & \multicolumn{1}{c}{ Motif } & Frequency \\
\hline AC & 93 & CG & 2 \\
ATC & 22 & AAAG & 1 \\
AG & 14 & AACG & 1 \\
AAG & 9 & ACGG & 1 \\
ACT & 5 & ACTGCT & 1 \\
ACC & 4 & ACTT & 1 \\
ACTG & 4 & AGA & 1 \\
ACAT & 3 & AGG & 1 \\
ATGT & 3 & AGGT & 1 \\
AAC & 2 & AGT & 1 \\
AAT & 2 & AGTC & 1 \\
ACACC & 2 & ATAA & 1 \\
AGC & 2 & ATAC & 1 \\
AGTG & 2 & ATAG & 1 \\
ATT & 2 & CGT & 1 \\
CCG & 2 & & \\
\hline
\end{tabular}

bers of the sequences submitted to the NCBI database. In total, 215 unique primers were designed with stringent conditions of $63^{\circ} \mathrm{C} \mathrm{Tm}, 3^{\prime}$-end GC clamp, and optimum length of $24 \mathrm{bp}$. Out of these 215 primer sets designed, we tested 192 primer sets on 16 DNA samples from eight $S$. frugiperda isofamilies. DNA extracted from individual fifth-instar larvae was used to test the 192 sets of primers. We report here the sequence information and screening results for 174 sequence-specific $S$. frugiperda SSRs that resulted in scorable PCR amplifications of the samples tested. The average length of amplicons for these markers was $134 \pm 25 \mathrm{bp}$. All forward and reverse primers had a Tm of $63 \pm 1^{\circ} \mathrm{C}$.

Marker Description and UPIC Scores. We used the UPIC software to identify the most informative markers obtained by screening $S$. frugiperda samples. Those with UPIC scores $>1$ from screening 15 DNA samples are listed in Table 4, zero would indicate that no sample is being discriminated by the marker. There were 17 markers with UPIC scores between 10 and 15, what means that those markers can uniquely discriminate between 10 and 15 out of the $15 \mathrm{~S}$. frugiperdaDNA samples tested.

Cluster Analysis and Genetic Variation of S. frugiperda. Insufficient DNA from one individual of isofamily 751 limited its fingerprinting to only 96 markers, so this sample was excluded from part of the general analysis. In total, 120 markers that amplified at least $93 \%$ of the samples were used to calculate genetic distances in a global cluster analysis for $15 \mathrm{~S}$. frugiperda DNA samples from eight isofamilies and three geographical origins, PR, TX, and MS. These markers detected 942 alleles with a range of one to 25 alleles per marker across samples, one to seven alleles per marker on individual samples, and an average of 1.4 allele per sample per marker. The average number of alleles per locus was $8 \pm 5$. In total, 103 of these selected markers had a maximum of two alleles on individual samples. Cluster analysis of the 120 SSR markers was performed. No phylogenetic analysis was intended from this preliminary screening, only the assessment of the usefulness of the markers. Pairs of samples from each 
Table 3. Microsatellite markers from S. frugiperda

\begin{tabular}{|c|c|c|}
\hline Marker & Forward primer $5^{\prime}=>3^{\prime}$ & Reverse primer $5^{\prime}=>3^{\prime}$ \\
\hline Stv_Spf20 & CCATCCTCAATGACAATTCCTATG & ATGTTGTCATCGTCTGTTGGCTAC \\
\hline Stv_Spf29 & TGATTGACGGACAAACATTCAAAC & TGGCAATAGGACTTTTTCCCATTAG \\
\hline Stv_Spf38 & ATTGCGAGATCTGTCAGTTTGTTC & AAATTCACGAATAACACGACAACG \\
\hline Stv_Spf61 & CCAAATTTATGCTACTGATGTACCTG & GGATCTATTGTTGTCGAAATACGG \\
\hline Stv_Spf69 & CCACCTCAACCACTGGTAACCTAC & ATTGACATGGAGTTAGGGTTTTGC \\
\hline Stv_Spf74 & TCGTTCTACGTGGAGGACCAAG & TTGCTTAATTAAACCTTACACGGAAGAC \\
\hline Stv_Spf85 & ACATCTTTCTTTGTTGACGTTTCG & GTATGTGATGGCTGTTTGTTTTGG \\
\hline Stv_Spfl02 & ACGTCGGGGTCACCACTGTAG & ATATACGATAGCCAGTCGGCTTCC \\
\hline Stv_Spf103 & CATACAGGACGTGGTGTAATGGC & GAAGCATACAGCCATACGTTACCC \\
\hline Stv_Spf120 & CATTGTACCAGTTGTGGGAGTTTG & AAATCAATCGTACCCTAAGTATTGCAG \\
\hline Stv_Spfl22 & AAGGGTAGCCTCCAAAGATAATCC & ACCTAGGTACCTTGACCCCAGAAG \\
\hline Stv_Spf127 & AAACATGACAAATATCCCGTTTCG & CTATGTTCAGCAGTGGACGTCTTG \\
\hline Stv_Spfl43 & GCGTTGTGTTCAACTGTTGTTATTAG & CTCTGCAGCACTGTAATAATGTCG \\
\hline Stv_Spfl47 & GGGTTGCGACTACTGATCTATGTACC & TAACTAAGCTCACGCAAACACTCG \\
\hline Stv_Spf148 & CCGTCATCTCACGTTCAGTCTAAG & TAAAATTGAGTAGCGTTTTTCGGG \\
\hline Stv_Spfl50 & TACTTACGCCATCACAACATCCAC & TCATCATCCTCTGACTCTTCATCG \\
\hline Stv_Spfl51 & TGGGGGTCACAGAACAGATAAAAG & GTAGTGGACTGTTAGGGGCAGTTG \\
\hline Stv_Spfl73 & TAACTTAGGCTGCAGACTCACCG & ATGTAGAGGAGGCTCGTAGTCCAG \\
\hline Stv_Spf187 & AGAATTTTGTGGAAGACAGGGAAC & GATAACCATAAGGGAGGGGGTG \\
\hline Stv_Spfl 88 & TGAGACGCATGGTGTTTAGTTTG & ATACACACCACACATCGCACC \\
\hline Stv_Spf212 & TGTGCGATGCAGGATATTTATGAC & GCGAGCATACTAAATACATCAAGGC \\
\hline Stv_Spf 240 & GCGGCAAACAATACCTGACATAAC & TTGTATGTTCCTTAGTGGGTTGGG \\
\hline Stv_Spf255 & GTGTAATCGGGACACATACAGCAG & ATGAGGATGGCTTCATCAAAGTTC \\
\hline Stv_Spf 270 & TGAACTGACAAACATATTGAATGCG & GTGTGTCCACTTTCCAAAACCTTC \\
\hline Stv_Spf292 & TGATAGACCGTGTGACTACCATGTG & TCATTACATCCAAAGCAACGTCAC \\
\hline Stv_Spf301 & GTATGTAAAGTGGAACAAACCGGC & TTGTATGTTTGTAAACGCACCCAC \\
\hline Stv_Spf305 & TTTGCAGTTGTGTCTGTGTTTGTG & AACTGTATGTGTGCATGCTGTGG \\
\hline Stv_Spf306 & CTCAACATACGCCCCGTCATC & ACCAGAGCGTTGTCGAAGTTACAC \\
\hline Stv_Spf343 & GTCAAAGTTTTACATGGAAGCGTG & CCCATCTGTTTGTCCACAGTAAAG \\
\hline Stv_Spf354 & GCCAGTCAACAAACACAGTTGC & GTTGTTGCTGTTGCTGGTGTTG \\
\hline Stv_Spf406 & TTAAGTCGTGCATAGATCCCGTAAC & TCGGCACTTGTATACGGAAATTG \\
\hline Stv_Spf407 & CAATAATTACCCGCGCATTATGTC & ACTCCTCCTTGTCCGTGTTGTG \\
\hline Stv_Spf413 & TAAAGATACATACGGAACGGAACCTG & ACAACTTACGTGTATCCCGGTTTG \\
\hline Stv_Spf417 & AACAACAGGGATAGATTGTGGACG & CACCAACTAACAACATCAGACCAATC \\
\hline Stv_Spf 452 & ATTGCTTCAGTACTCAGTCGGCTC & ACACGATACATTGTACACGATGGC \\
\hline Stv_Spf462 & CCTAACCAAAGAACATGCACACAC & ACGTTGTTATTGGTGGGATAGCTG \\
\hline Stv ${ }^{-}$Spf 467 & CACTGTTAACGTTTGAAGTGGGTG & TTCAAACAGCGCTCAATACAACTG \\
\hline Stv_Spf 470 & CAACTAGCTCTGCGCGCACTATAC & GGGCTACACTCCAACTTTACCGAC \\
\hline Stv_Spf488 & ACATCGAAAGGAGATGAGTTGTCC & GTATGGTCATTCGTAGCTTACGCC \\
\hline Stv_Spf526 & GAACAAAAACAGGCGTGAATATGG & CGAATGTTGTTGACTCCGTATGAC \\
\hline Stv_Spf538 & TGATAAAGGTGATATGTGCTGGGG & ACTCCACAGCAAGTCAACACACAG \\
\hline Stv_Spf544 & TAAAGCAAATCAAACAATTTGGGC & AGTGTACACAGATATGGAAGGACGC \\
\hline Stv ${ }^{-}$Spf552 & TCACTCCGTACATCATTTCTCAGC & ATCGCCATTTAACTAACGACCATC \\
\hline Stv_Spf559 & CAAGGTGTAGTTCGGAGGTAAACG & CGAACCAGTTGTCATTCTCCATC \\
\hline Stv_Spf578 & TGCAAAAACATTTCCTCAATAACTG & AGTACGGTTGATTTAAGATCGCTATG \\
\hline Stv_Spf581 & GTTTCCTTAAAAAGATCGCGTGC & GCTTCTTTGTTGAGAGTTGAAGCC \\
\hline Stv_Spf587 & CCATAATCGACACCGATTGCTTAC & CGATTATTGATTAGGAATACGAAGATGG \\
\hline Stv_Spf606_a & CTTAGGTGGGACCATTTTCTTTGC & AGATACGCTGAAAGCCTAAGGGAC \\
\hline Stv_Spf606_b & CGTGATGTCGTATATAAGGTCGGG & AAGGAGATACGTGGAGTTGTGACG \\
\hline Stv_Spf615 & ATTCCCCCAGAGACGATGAAGTAG & TACAGTGAAATGGGTTGATGTTGG \\
\hline Stv_Spf636 & TATCGATTCCTCCCACACACCTAC & TGGGTGTATGTCCTCTTTTTGTCC \\
\hline Stv_Spf653 & TTGAGTGTTTGTTCAATTCATGGG & CGCTTCAAACATTCACTCACAAAC \\
\hline Stv Spf658 & TTAAACACCTCACACACCTTGCTG & AACCACGAATCCTTCCAATACACC \\
\hline Stv_Spf662 & TATCATTATCATGAGTTGTCCCGC & ATTGAACAGTTTACTTCGGACGTG \\
\hline Stv_Spf664 & AGCCAAGTAACATGACGAGTAAGC & TGATAATACAATTAAAACCGGTATTGTG \\
\hline Stv_Spf670 & GGGAGAGGTTTCTAGCTTCTACGG & GAGGAGCCTTGGTTCAATAGTGC \\
\hline Stv_Spf686 & CAAGGACATCCTTCCTTGCAGTAG & ACTCACTCACTCACTCACTCGCTC \\
\hline Stv_Spf688 & CTGCCTAATACTCTGCTTCATCCC & TACTTGAACAAGTGGCAGAGCAAC \\
\hline Stv_Spf692 & TGTGACGTCATCTCACAGTCTCG & GATACCAAAACCGAAGCACACAAC \\
\hline Stv_Spf717 & AGGGTCGTTTTCAGGGTTTTAGAG & CCACGAAGTCTACCACATCTACCC \\
\hline Stv_Spf728 & ACTTCGATTGTGGAACTCTTGACC & ACTGTTAATGCTGAGCAAGAAGCC \\
\hline Stv_Spf738 & AATTGAAAGAATTTCCGTGTGTGG & ACGACAGACCGTGAAAATAACTCC \\
\hline Stv_Spf743 & CGAGCAGAGTGAATATGAGAGGTG & TCCAGCTGTGTCTCCATTAATATACG \\
\hline Stv_Spf746 & TATTTCAGACCGATCTGTCCAGTG & GCTTGCTGTGATTAAGCCGATAAC \\
\hline Stv Spf747 & ACGAGGATGATGATGAAGAAGGTC & ATTATCAGCATCACGATCGCCTAC \\
\hline Stv_Spf751 & GTAAATAAGTCATTGGTCAAAAGCCC & CATGACGAATCTATAAGGGTTCCG \\
\hline Stv_Spf752 & TGTTCTATTGTCTCTGCCTACCCC & TGTGGGCTCAATTTGCATACATAC \\
\hline Stv_Spf756 & TGCCGATACCTAGAGTTCCGAG & GAATAGGTGTAATCAGATGGTGCG \\
\hline
\end{tabular}


Table 3. Continued

\begin{tabular}{|c|c|c|}
\hline Marker & Forward primer $5^{\prime}=>3^{\prime}$ & Reverse primer $5^{\prime}=>3^{\prime}$ \\
\hline Stv Spf764 & TAGGTTCCTCTATTGGGTGCAGTC & CGAGCTATCATCTGCCTCATTACC \\
\hline Stv_Spf783 & TGCCATATTATACTGGGCACAATTC & TGCGTTGTTGGTTTAGTGTTCAAG \\
\hline Stv_Spf789 & CGACACGTTGATTGCTCACAG & AATCTTTTATCACAATTCGCAGCC \\
\hline Stv_Spf807 & CAGACGATAGCATGTGTCGATGTAG & TCGATAATTTCTGCCTCACCAATC \\
\hline Stv ${ }^{-}$Spf 823 & GATCAATGTTCAAACCTTCTCGGT & CTAACGCTCTACGCTTGACGAAAT \\
\hline Stv_Spf824 & CGTGCTCATTAGCCTGTTTAATTTTC & GGGTGTGTCGTGGTCCTATTTTATC \\
\hline Stv Spf828 & CCATGTAGCGTAACATACAGCACTC & AAACCAATTCAACAGAGCGTCTTC \\
\hline Stv_Spf835 & AAATGTGGAACACCTTTGTCTTGG & CTGCGATAATTACAAATCAACAAAACAC \\
\hline Stv_Spf858 & CTGCAAGGAGAGCACCACTGTC & CAAATGGGACGACATTCGCTAC \\
\hline Stv ${ }^{-}$Spf 868 & TTGTTGTAATTGAAAAGATGGCCG & CCTTCAAAACCAAATTTGAATGTATCC \\
\hline Stv ${ }^{-}$Spf869 & CGTTCTAATTTATCGCACCATTCAC & TGATCGACGTTTACGAAACTTATGG \\
\hline Stv_Spf904 & TATGTCCTGTCGCCTGTCATACAC & TTCTGAAATGAAATGGATTTTCGG \\
\hline Stv_Spf908 & AGTCATTTACAACAAGCTGGAACG & GCATCTAACTTGAACATTTTCCCC \\
\hline Stv_Spf914 & TCCAAATTCAAATTTCATTCCACA & TAGTGTTTGAGCAGCTCTGCTTGT \\
\hline Stv_Spf918 & GCGAAATTGTTTTAATGTGGGTTG & ACGACCTATACGGACCTTGTTACG \\
\hline Stv_Spf929 & TTATTTACGCGGGAATCGTTTATG & ACTACATAAATTCGCACAATCCCG \\
\hline Stv_Spf941 & GAATCTCGGCGAAACAAGGTTAC & ATTATTGTTTGTTTCATCGTCGCC \\
\hline Stv_Spf950 & ATGATATCGTCTGATGCTGACCAC & ATGAGGGTGATTCGAAAAATCTTG \\
\hline Stv_Spf967 & GTTCGATCGGTTTCGAGTAATGTT & CACTCACACCCATAAAACCATTCA \\
\hline Stv_Spf975 & GCAGTGATGAGAGATTAATGTGCG & TTTTTGTCGGATGTGTGTACGTG \\
\hline Stv_Spf978 & GGACATCTGTGGTCAGGATAGCTC & TGTCTCCCAAGGTTTTAAGTCCAC \\
\hline Stv Spf988 & TGTTGGGTATTGTGTGTAGTTTTGG & CTGACTAAAAACCAACCACGTTCC \\
\hline Stv ${ }^{-}$Spf994 & CCCTCTTTAATGAAACGGAGTGC & CTTAGTAACAGCGAGGACGTCAGG \\
\hline Stv Spf997 & TTGATGCATGAATTTTCAAACGAG & ATCACGTTGTGGTCCAATCAATG \\
\hline Stv_Spf1000 & TCGACGAGAGGTTTGTTTAGTTCA & AAAACCACAACGCAAGTAACATGA \\
\hline Stv_Spf1036 & GTGTTAGTAATCTGTGGCTGTGCG & CTAACAAACACTGAGGAGGCAAGTC \\
\hline Stv_Spf1050 & CGGCGAAGTAGGACATAGAGTGAG & CGACATCAAGCAGCATTAGTTCAG \\
\hline Stv_Spf1057 & TGTAATGTTCCATGTAATGGGAGG & TCTCTGCCTACACTCATAGGCTTG \\
\hline Stv_Spf1058 & AAACTTTCACATGATTTGTTGACG & TCGTTCATACAAAACATAACAACAAAC \\
\hline Stv_Spf1068 & CGACACGTAATGCTGCTATACAATG & CGAAGTATTACGGGGCATTTTTC \\
\hline Stv_Spf1075 & TCTCTGGTGGAAAAGCATCTATAAC & CACAAACCACCAAGTCTTGTACTG \\
\hline Stv_Spf1079 & AATAACATGATTCAAGGCTACGGC & CGTATAGGGGAATAATCTCGCTTG \\
\hline Stv_Spf1098 & CATCTAAATCCGAACCGATGAGAC & GCAATGACAGATGCATTATAAATACACG \\
\hline Stv Spf1102 & CAAACCTTCTCCGTATGAAAAGAG & AAAAGACATAAAACTAACTGTAGCACCC \\
\hline Stv ${ }^{-}$Spf1106 & CGAAACAAGTGAATCTGTCAGTGC & ATCATCACCATCATCATCACCATC \\
\hline Stv ${ }^{-}$spf1120 & AACAACAAGTGAGTATTTTCACACAGTC & CAATAACTTCTTTGAAGGTGCTGC \\
\hline Stv_Spf1125 & GGCTCTTGGGGGTTATGTAAGAAG & GGTTCCAAACTACCACGGATAATG \\
\hline Stv ${ }^{-}$Spfl1128 & ATACATAACCTCACGCCTGTCTCC & TTCTTGTCGTAGTAGTAGCGCAGC \\
\hline Stv Spf1134 & GTGGTTGGAGACTGTACCGGAG & CTTGTCTCAGTCTTGGGACTCCAC \\
\hline Stv Spfl136 & ATTACCTCGCACTCAATTAGCCAC & ATAATCACACACCGCCTCAGTACC \\
\hline Stv ${ }^{-}$Spf1147 & AAACTAATAAACAGAGTTGCTCCATCC & CTCTTGGCGGATAAAAGGTGTAAC \\
\hline Stv_Spf1170 & AATTGCCGGAATTAGACACTTCTG & AACCGTTCCGGTAAAGTATATGGG \\
\hline Stv Spf1171 & TTGAACGGGTACGTATTACCAACA & ATAATGGTGACGTGCCATTGAAG \\
\hline Stv_Spf1176 & TTATACCGATATTCCGGTCCAGTC & ACAGCAATATATCTTATCTACGAGCCTG \\
\hline Stv ${ }^{-}$Spf1195 & GTCGCCATTATTATGGACCTGAAC & GATTTGACTTTGACTTTGAAGGGC \\
\hline Stv Spf1221 & TTAGTCCAGTGAACAACTGAGGGAG & CGACGATGAACCTGAAATGATTC \\
\hline Stv_Spf1230 & TACAAGGTTCCGGCTTGAAAAATA & CCGTCGCTAGTGATAGAGCAAAGT \\
\hline Stv_Spf1231 & TCAACCGACCAATAGTGATGACAC & AGTTACAAGATTTCACGTCACACACC \\
\hline Stv Spf1243 & GCTTCATCAAGACGGGAGACAG & GACGTTTATTCGGTTATGGGACAG \\
\hline Stv_Spf1245 & TCTTGGCTTAGATGTGGATTTAGG & TCTGTCATGATACCTTTATCAGTTTTTG \\
\hline Stv_Spf1260 & CACGATCTCCACATGGAACTAGG & ATTGTTGTAAACATCGGCTATCCC \\
\hline Stv Spf1264 & AAGTTGAGGTCTTTTGGTGACGAG & ATTTTCGGAAGCAATTACTAGGGG \\
\hline Stv_Spf1268 & ATTGACCAGACCTTGCTAAAATGG & GCTGCTCGGTTGTATATTTGTGTG \\
\hline Stv_Spf1279 & AAGCAAATAAACACGTTCACAAGC & CGCTCTCAAAGTTAAGAAATGTATGTG \\
\hline Stv Spf1304 & TTCAGTAATGGGATGGTTACAGGC & ATTGATGGGCAAATTTGAGAGAAC \\
\hline Stv_Spf1315 & AGATGTGGAGGTGTTAGTGTGTGC & ACCTCACCCATACCAAATCGAAC \\
\hline Stv_Spfl334 & TTCTGCTTGGAATTCATACAGTAACAC & TTTTACATCCCTCAACGTCTCACTC \\
\hline Stv Spf1349 & TTAATCAAGATTTCCTGCCACACC & GGCTTTTGCTTAACAAACAGAGAGG \\
\hline Stv_Spfl363 & TACCCGGTAGTCGCTTACCCTAAC & ATCGTATTGTGCAGATGGTTTGTG \\
\hline Stv_Spf1382 & TACAGGAAGGTTCATAACTGTGGC & GGTCCGACCTACGTACACTCAAC \\
\hline Stv_Spf1387 & CAGTGGACTGCTATAGGCTGATGA & CAAGTTTACCGATAACCAAAGCCA \\
\hline Stv_Spf1396 & CATGCATGTCTCTTTGCTGTATG & GCAATCTTCTCGTTCAATCAATG \\
\hline Stv_Spf1401 & GTTGCAAGATTCCACTTCACACAC & CGAAAATGTCACTTAATGCACCAG \\
\hline Stv Spf1406 & TTCATGCAGATTGACGAAAATGAC & TTACGGGCCTAAGCCAAGTAAAG \\
\hline Stv_Spf1409 & TAATGACGCGTAAATTATTCGCAC & TAATCCCATTCAACGTCACTCATC \\
\hline Stv_Spf1419 & CCTCAATATAACTCCCGAAGACCAC & TTTCCGATATTTCGGCACTGTTAG \\
\hline Stv Spf1432 & CCAAATAATTGCACAATACCTGCAC & TCGATAGTTGCTCACATTTGAAGG \\
\hline Stv_Spf1435 & GGGTCTTCACTTCACAAGCACATAC & TGTCAGTTTAACTTTAATTCGTCGGC \\
\hline
\end{tabular}


Table 3. Continued

\begin{tabular}{|c|c|c|}
\hline Marker & Forward primer $5^{\prime}=>3^{\prime}$ & Reverse primer $5^{\prime}=>3^{\prime}$ \\
\hline Stv_Spf1447 & TGTAAAGAGGTCAATTGGCATGAG & ACTGCACTGAACACGGTGATTAAC \\
\hline Stv_Spf1460 & ACAGTGACTGGGATGAAGAGCTG & AGGCTTATTATACTACGTGCTCACCAG \\
\hline Stv_Spf1461 & CCGGGTCTGTCAAAGTATTACTGG & TTGCCATATACTGGACACAATTCC \\
\hline Stv_Spf1464 & AGCTATAAACACAGGCTCCTCGTC & TAAGAATCAAGCATACAATTGGGG \\
\hline Stv_Spf1466 & GTTTCACGTGCCCCAACTACATAC & AGTACGTTTCTGTACCAACGGCTC \\
\hline Stv_Spf1471 & TCAGTAGTGGTCACTGTTGAAGTGG & TCCCGGGATTAGAAATATTTACCG \\
\hline Stv_Spfl473 & GTTTCATTCACTCGCCACACTG & GGCTGCGTAGTTTAAACAATTAAC \\
\hline Stv_Spfl486 & GCGGTAGTACCAAGGTGAGGTTAC & ATAAATGAAACGTTCACAACACCG \\
\hline Stv_Spf1502 & TTTGCAATTTTAGTTACAAACGTCCTC & TATTGATAGCCTCGTGTTTGACCC \\
\hline Stv_Spf1539 & TTGTAGGGGCATGATTATTGAAGG & TTTTTGAGGAGGGAAAACTCGC \\
\hline Stv_Spf1552_a & CAAGGGGTGGGGAATAATTAAG & CCACACCTTGCTTACTTTCACTTAC \\
\hline Stv_Spfl552_b & AAGTGAAAGTAAGCAAGGTGTGGG & TTTGCTCACTCACTCATTCATTCTG \\
\hline Stv_Spf1561 & ATATCAAGATGGGCTACAAAACCG & CTTTCCAGTTGGAACGAACTTACG \\
\hline Stv_Spfl573 & CAATAGGAGAAAGGCGTGAATTTG & TCGTTAGAACTAGCCATTTGGAGC \\
\hline Stv_Spf1576 & CACTAATTACCAGTCACCCTATGCG & TAGCTTAGCTGAGTCCGTTTCCAC \\
\hline Stv_Spf1582 & AAATATTTGCTCGGAGGTATATGGC & AAAACCTTGTCTCCCTTCCACTTC \\
\hline Stv_Spf1587 & TCGTACAAAACCTGCTTAAACTTGG & TCGGACGAACTTTGTGTACGTG \\
\hline Stv_Spf1592 & GGTTCCTGTTATCACCTGCAGTA & CTATGTAGTTTATGTTAATTCGCACGAT \\
\hline Stv_Spfl600 & AAAGTTCCGACGGACAACTTGTAG & AATCGATCGTGTTTGTGTTGTCC \\
\hline Stv_Spfl604 & GGAGGTGTGTATTATCATGTGTTTGG & TTTGTTCCGAAAAATTCGTTAATTG \\
\hline Stv_Spf1634 & TGTAACACTTGATGATAATGCGCC & CGATATTCGCTTACTTGGAAGGTG \\
\hline Stv_Spf1651 & ACATGGAATTGTTTGTTTAATGAAAGG & CATTTAAGACAAGAGACGCGCTG \\
\hline Stv_Spfl673 & TGGCTTTTAGTCAGTAAATGGCTG & AGGCACACAATTCCCTTTTTGTAG \\
\hline Stv_Spf1680 & GGTTGGCATTTGAATAATTCTTTTTG & AGTCTGATGACTCTTTGAGCGGAC \\
\hline Stv_Spf1683 & TCATCAGGGAAAGGAGTTGAACAT & AAAGCTAGGTGTTGTGAGACTCGG \\
\hline Stv_Spfl685 & ATAACTGGCTATTTGCCGTATTGC & AAAGACAAGTTCAAAGTTCACCAACAC \\
\hline Stv_Spf1690 & ACTAGGATTTTTGACGGTGTGTCG & CATTCGATAGTTGACTCGCTTTGG \\
\hline Stv_Spfl698 & CGCATGCTGACCCTAAGTCTTTAC & GACTGTAGGCGGTTGATGTGTG \\
\hline Stv_Spf1706 & CCACTGTACTGTGATAAACAGATGGC & ATGATCATACAAAGTGCATCCGTG \\
\hline Stv_Spfl707 & AAAACATCAAACCTATTCAACGCC & CCTTCACGTCATTCAGCAAACTAC \\
\hline Stv_Spf1712 & AACACAAATTACACCAACCAAGCC & TTTTAGCGCCTCGAGATTGTTATC \\
\hline Stv_Spfl713 & TAAAATACTTATTACGCGAAGCCG & AAGTAAGACACGAATGTATCAATTTTCC \\
\hline Stv_Spfl723 & TTTGTTTAGCAATGGACGTCTCTC & ATCGTGTCTGGTGCTGAATAAATG \\
\hline Stv_Spfl728 & TATGTAGGCAAGGTAACCGGACTC & CGTGTACTCGTTTCCCAACCTATC \\
\hline Stv_Spf1747 & TGTAATTTTGTGTTTGTGTTGTGCC & AGGTCTGTCTAGGTAGCGAGCATC \\
\hline Stv_Spfl758 & TCACACCACACCATATCACAACAC & TGTATTTGTAAGTTTGACCGAGTGAG \\
\hline Stv_Spf1783 & CAAGCGTACATCGAGTCAAGGAG & GCAAATTAATCCCGCACTGTTTAC \\
\hline Stv_Spf1844 & CTACCCCTTCGGGGATAAAAAG & TTGAGTCTCTTTGAGTAAAACCGC \\
\hline Stv_Spf1856 & TCATCATGGAGATTACCTGGACTG & AGTCACACAGTCAGCCACAAAAAC \\
\hline Stv_Spf1860 & AATGTTGAGAAGTTCTGCCTACCG & ATCTACCTGCATGCCAAATTTCAG \\
\hline Stv_Spf1863 & AGGGGGTCTTATTAAAGTGGTGGG & ACCATGACACAACGAGGCCTATAC \\
\hline Stv_Spf1890 & CATCTCATTTTTGAAGAGATCCCG & TTCGCGTGGAATAGTGACTTCC \\
\hline
\end{tabular}

Marker numbers correspond to the contig names of the sequences submitted to GenBank, NCBI. Max alleles/sample, maximal number of alleles observed on individual samples; $\mathrm{N}_{\mathrm{o}}$, total number of alleles observed for that marker; $\mathrm{N}_{\mathrm{e}}$, effective number of alleles; $\mathrm{H}_{\mathrm{E}}$, expected heterozygosity; $\mathrm{H}_{\mathrm{o}}$, observed heterozygosity; Hits, $\mathrm{N}$ indicates BLASTn expected values of $1.0 \times \mathrm{E}^{-04}$ or lower, and $\mathrm{X}$ indicates BLASTx expected values of $1.0 \times \mathrm{E}^{-04}$ or lower; asterisk $(*)$, markers used for cluster analysis in Fig. 1.

population were grouped together by the cluster analysis with a maximum genetic distance of 0.56 (dotted line) within colony, and the minimum genetic distance among colonies was 0.83 (Fig. 1, dashed line). Bootstrap coefficients higher than $50 \%$ are shown at the nodes in the dendrogram (Fig. 1). High reliability of the clusters was estimated for the clades at the colony level by bootstrap values of $100 \%$.

Shared Alleles Among Geographical Locations. For the 120 microsatellite markers that amplified across samples resulting in 930 alleles detected, we summarized the number of alleles present in at least one individual of each geographic area and represented the results in Fig. 2. Despite the limited number of samples analyzed, $\approx 100$ more alleles were found in the colonies from TX than in PR and MS, and the percentage of alleles of TX that also were present in PR and MS was the lowest (between 34 and 39\%). Only $14 \%$ (127) of the alleles were present in at least one individual of each geographical area.
BLAST Results. DNA sequences obtained from the microsatellite-enriched libraries of S. frugiperda were masked for repeats and screened using BLASTx and BLASTn and the results are shown in Supp. Table S1 [online only]. Markers for which their corresponding sequences had significant hits were indicated in Table 3. Markers for which sequences had expected values of $1.0 \times \mathrm{E}^{-04}$ to $1.0 \times \mathrm{E}^{-38}$ for BLASTn and $1.0 \times \mathrm{E}^{-04}$ to $1.0 \times \mathrm{E}^{-144}$ for BLASTx are shown in Table 3, using a letter $\mathrm{N}$ for significant BLASTn hits and a letter $\mathrm{X}$ for significant BLASTx hits.

\section{Discussion}

Microsatellites for S. frugiperda. Microsatellites are desirable over many other molecular markers for being highly polymorphic, highly abundant, codominantly inherited, simple to analyze, and readily transferable (Weber 1990). The microsatellites presented here are not only sequence specific for S. frugiperda 
Table 4. List of markers with UPIC scores different from zero, calculated for S. frugiperda microsatellite markers using the UPIC software (Arias et al. 2009)

\begin{tabular}{|c|c|c|c|c|c|c|c|}
\hline Marker & UPIC Score & Marker & UPIC Score & Marker & UPIC Score & Marker & UPIC Score \\
\hline Stv_Spf01128 & 15 & Stv_Spf01747 & 9 & Stv_Spf01231 & 7 & Stv_Spf01079 & 4 \\
\hline Stv_Spf00670 & 13 & Stv_Spf01409 & 9 & Stv_Spf00147 & 7 & Stv_Spf00728 & 4 \\
\hline Stv_Spf00997 & 13 & Stv_Spf01401 & 9 & Stv_Spf00301 & 7 & Stv_Spf01268 & 4 \\
\hline Stv Spf00789 & 13 & Stv Spf00692 & 9 & Stv Spf00858 & 7 & Stv Spf01279 & 4 \\
\hline Stv_Spf01592 & 13 & Stv_Spf00751 & 9 & Stv_Spf01382 & 7 & Stv_Spf00187 & 4 \\
\hline Stv_Spf01706 & 13 & Stv_Spf00994 & 9 & Stv_Spf01195 & 7 & Stv_Spf01576 & 3 \\
\hline Stv_Spf00918 & 13 & Stv_Spf00103 & 9 & Stv_Spf01683 & 7 & Stv_Spf01106 & 3 \\
\hline Stv_Spf00343 & 11 & Stv_Spf00578 & 9 & Stv_Spf00452 & 6 & Stv_Spf00747 & 3 \\
\hline Stv_Spf00658 & 11 & Stv_Spf00240 & 9 & Stv_Spf01102 & 6 & Stv_Spf00462 & 3 \\
\hline Stv Spf00544 & 11 & Stv Spf00606 b & 9 & Stv Spf01334 & 6 & Stv Spf01260 & 2 \\
\hline Stv_Spf01486 & 11 & Stv_Spf01419 & 8 & Stv_Spf00908 & 6 & Stv_Spf00606_a & 2 \\
\hline Stv_Spf01856 & 11 & Stv_Spf00746 & 8 & Stv_Spf00752 & 5 & Stv_Spf00212 & 2 \\
\hline Stv_Spf01502 & 11 & Stv_Spf01432 & 8 & Stv_Spf00173 & 5 & Stv_Spf00835 & 2 \\
\hline Stv_Spf00552 & 11 & Stv_Spf01651 & 8 & Stv_Spf01447 & 5 & Stv_Spf00653 & 1 \\
\hline Stv_Spf01587 & 10 & Stv_Spf01698 & 8 & Stv_Spf00764 & 5 & Stv_Spf00255 & 1 \\
\hline Stv_Spf01844 & 10 & Stv_Spf01707 & 7 & Stv_Spf00120 & 5 & Stv_Spf00305 & 1 \\
\hline Stv_Spf00406 & 10 & Stv_Spf00292 & 7 & Stv_Spf01396 & 5 & Stv_Spf01890 & 1 \\
\hline Stv_Spf01685 & 9 & Stv_Spf01783 & 7 & Stv_Spf01758 & 5 & Stv_Spf00467 & 1 \\
\hline Stv_Spf00914 & 9 & Stv_Spf01723 & 7 & Stv_Spf00581 & 5 & Stv_Spf00150 & 1 \\
\hline Stv_Spf01264 & 9 & Stv_Spf00417 & 7 & Stv_Spf00538 & 4 & Stv_Spf01245 & 1 \\
\hline
\end{tabular}

but also were designed using stringent conditions regarding runs, overlapping with repeats, 3'GC clamp and a uniform high melting temperature $\left(63 \pm 1^{\circ} \mathrm{C}\right)$ to minimize the need of PCR optimization. Until now, microsatellites have not been reported for $S$. frugiperda, and there are only eight microsatellites re-

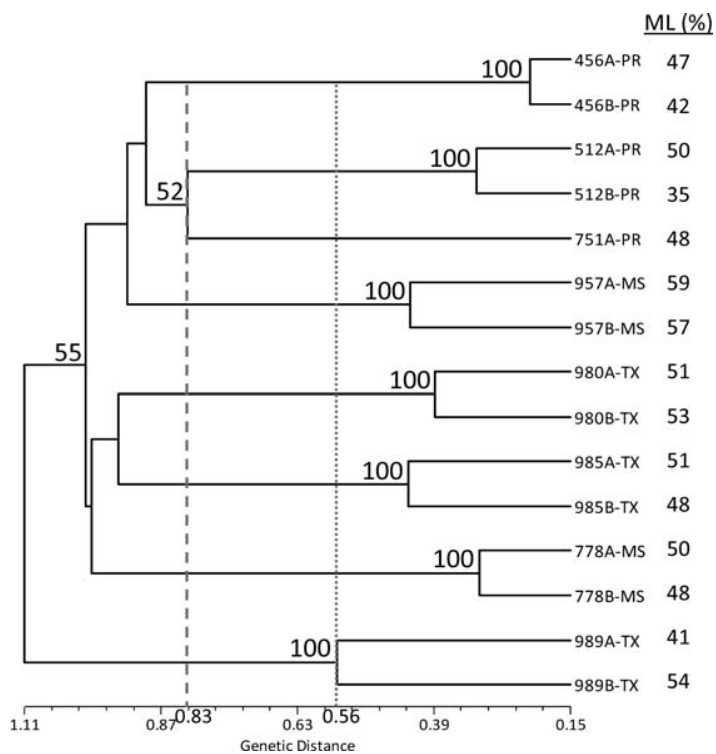

Fig. 1. Cluster analysis of 15 individuals of eight S. frugiperda colonies by using 120 microsatellite markers by the unweighted paired group method and arithmetic averages (unweighted pair-group method with arithmetic average). Genetic distances were calculated with Nei's coefficient, and cluster analysis by the algorithm implemented in the SAHN program of NTSYSpc version 2.2. Bootstrap coefficients higher than $50 \%$ after 5,000 resampling are shown at the nodes. ML (percentages), multiallelic loci in percentage, represents the percentage of loci that showed multiple amplicons. ported for a related species, Spodoptera exempta (Walker) (Ibrahim et al. 2004). In the present work we have contributed to the NCBI database with 187 nucleotide sequences of $S$. frugiperda microsatellites from where the markers were isolated. In total, 125 of these microsatellite markers amplified across the samples and 103 of those markers presented no $>2$ alleles per sample, making them ideal for population studies.

UPIC Scores. The discriminating power of the markers was evaluated by calculating the UPIC scores. UPIC score of a marker represents the number of DNA samples that can be individually discriminated from the rest of the samples by that particular marker, i.e., a UPIC value of 2 indicates that two samples have unique allele patterns for that marker and can be

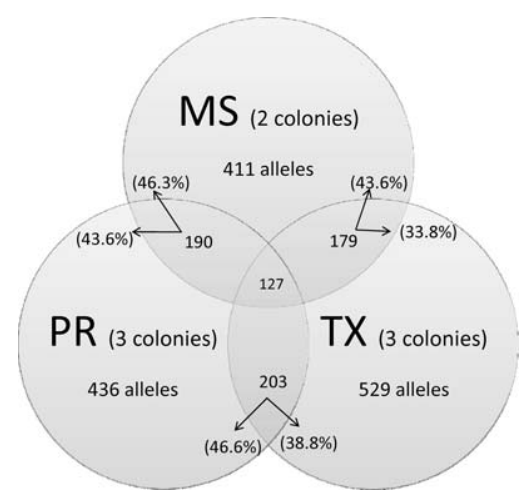

Fig. 2. Alleles shared by S. frugiperda individuals from three geographic areas, Puerto Rico (PR), Texas (TX), and Mississippi (MS), in the analysis of 120 S. frugiperda specific microsatellite markers. The Ben diagram shows the total number of alleles observed in individuals of each region. Numbers on the overlapping areas of the diagram correspond to the number of alleles observed in at least one individual of each of the geographic regions. Percentages were calculated based on the number of alleles shared and the total number of alleles observed in each group of samples. 
distinguished from each other and from the rest of the samples (Arias et al. 2009). UPIC values listed here can be used to plan future experiments, allowing the selection of the most powerful discriminant SSR markers, which are those with the highest UPIC score, and thus reducing the cost of the analyses. For example, the top markers with the highest UPIC scores (Table 4) will provide the maximum discrimination of the genetic variation within and among $S$. frugiperda populations.

Isoline Identification. Two S. frugiperda biotypes have been identified so far, the rice and maize biotypes (Pashley et al. 1985, Pashley 1986). Though these biotypes present no morphological differences, they are effectively distinguished by molecular markers such as the Tpi gene (Nagoshi 2010), FR (Lu et al. 1994), the COI gene (Pashley 1989), and AFLPs (Prowell et al. 2004). However, there is evidence of interstrain matings (Nagoshi and Meagher 2003), and in some cases the molecular markers available did not distinguish genetic differences among populations from distant geographical areas, such as Brazil and North America (Machado et al. 2008). Although we do not attempt to make any phylogenetic inferences from the cluster analysis, we do point out that each population was discriminated with bootstrap values of $100 \%$. This indicates that these microsatellite markers will significantly contribute to the pool of molecular markers available for $S$. frugiperda as a large number of loci (174) were analyzed in this work. Such a resource can be used to address issues of biotype, migration, and phylogenetics. The microsatellites reported here can distinguish isofamilies 456 and 512 from each other and from the rest of the colonies; 456 and 512 share geographic origin and present resistance to Bt Cry1F and CrylAc proteins (Blanco et al. 2010). Further testing comparing susceptible and Bt-resistant strains from the same geographical origin would be necessary to test the use of these markers in resistance monitoring. Thus, the markers provide a useful tool to differentiate isofamilies (Fig. 1).

Allele Overlapping on Geographic Regions. One advantage of the availability of a large number of molecular markers is, that while studying many individuals at few loci is useful to infer recent population history, a large number of loci are more likely to contain information about older events (Wang and Hey 2010). Using mitochondrial haplotypes, it has been reported that $S$. frugiperda populations of Mississippi and Florida were similar to the haplotype profile found in Texas (Nagoshi et al. 2008). In our cluster analysis, one of the two MS populations analyzed by SSRs did group close to two TX populations, whereas the other MS population grouped with the populations from PR (Fig. 1). The low resolution at the base of the dendrogram indicates that possible crosses may have already taken place between MS and PR populations and between MS and TX populations. In addition, the number of alleles shown in Fig. 2 for individuals of the three geographic regions, PR, TX, and MS, showed that between 39 and $47 \%$ of the alleles are shared between geographic regions and only $14 \%$ of the alleles were observed in all three regions. The larger number of total alleles in TX and lower percentage of alleles shared with other regions, point to a potentially higher genetic diversity in this region. Having the availability of the markers presented here, it will be feasible to analyze a much larger number of samples from distant areas to better understand the potential migrations and crosses of this insect, and this would influence the management practices.

BLASTn Screening of Marker Sequences. Among the first 125 markers reported in Table 3, several interesting hits were detected using BLASTn. For example, Stv Spf29 had homology to a gallerimycin, Stv Spf662 to Serpin-1 alternative splicing isoform, Stv_Spf581 to the ITS 18S ribosomal DNA, Stv_Spf1230 to a chitinase, Stv Spf301 and Stv Spfl844 to chitin synthases, Stv Spfin068 and Stv_Spfil461 to alpha-amylases, Stv_Spf869 to a juvenile hormone, Stv_Spf38 Stv_Spfl 27 and Stv_Spf1419 to signal transducers, Stv_Spf07 477 to a transcription factor, Stv_Spf1230 to a chitinase, Stv_Spf1890 to a nucleopolyhedrovirus mutant, and Stv_Spf417 Stv_Spf746 to cytochromes P450. The potential impact that could result from the genetic polymorphism on the mentioned DNA sequences of $S$. frugiperda is for the most part self-explanatory. We will only add that gallerimycin is an antifungal insect defensin shown to be induced by Beauveria bassiana (Bals.-Criv.)Vuill. in Samia cynthia (Druri) (Hashimoto et al. 2008), whereas Serpin-1 is a serine-protease inhibitor (Potempa et al. 1994), known to be involved in insect-immune response and to present exon expansion in Lepidoptera (Hegedus et al. 2008). Other sequences with non intuitive function would be those of $\alpha$-amylases, but these enzymes can be activated up to five times in S. frugiperda during larva feeding (Lwalaba et al. 2010).

MarkerStv_Spf746 is very interesting because it had similarity to the antifreeze protein Lu-1 of Helicoverpa sp. At this locus (marker Stv_Spf746), whereas all PR samples had the same unique allele, samples from MS and TX had 50 and $100 \%$ heterozygosity and a total number of 18 alleles. In the freeze-intolerant moth Choristoneura fumiferana (Lederer), the expression of several isoforms of antifreeze proteins serves to this insect as protection for overwintering (Doucet et al. 2002). Considering that in Puerto Rico there are no freezing temperatures and only one allele was detected in samples from that island, the large polymorphism observed at this locus across S. frugiperda samples from continental United States suggests a possible biological meaning for the genetic variation observed. S. frugiperda is believed to overwinter in the southern U.S. states, becoming source of infestation in northern areas (Nagoshi et al. 2009).

Another interesting marker was Stv Spf173 that had homology to an EST of antennae tissue of Spodoptera littoralis (Boisduval), part of an olfactory and pheromone detection resource, uploaded to NCBI (EZ981883.1) by E. Jacquin (INRA, Versailles, France). The allele distribution of Stv_Spf173 allowed unique identification of each of the eight colonies tested. Because the use of pheromones is essential for pest control (Witzgall et al. 2010), and the 
evolution of pheromones in Lepidoptera has an important role in insect speciation (Roelofs and Rooney 2003), the genetic variability at this locus makes us wonder how much more we need to know about the genetics of $S$. frugiperda to develop effective methods of control. Probably, the first target of future work will be to find out whether an association exists between these last two markers Stv Spf173 and Stv Spf746, and either cold tolerance or colony recognition in $S$. frugiperda.

BLASTx Screening of Marker Sequences. Also, among the first 125 markers shown in Table 3, significant similarity $\left(\mathrm{E}\right.$ value $\left.\leq 1 \times \mathrm{E}^{-04}\right)$ existed between several sequences and protein databases at NCBI. For example, Stv Spf255 had similarity to a cadherin, Stv Spf292 to an antennal esterase, Stv Spf1221 to a calnexin, Stv Spf686 to the stomato-gastric nerve plexus protein of Apis mellifera (L.), Stv Spf743 to a peroxisomal biogenesis house-keeping protein, Stv Spf1245 to an x-ray repair protein, and Stv Spf69 Stv ${ }^{-}$Spf122 and Stv Spf1582 to reverse transcriptases. Although the potential effect of genetic variation on these proteins might be self-explanatory, we want to indicate that cadherins are transmembrane proteins known as receptors of the $B$. thuringiensis toxins in Lepidoptera (Vadlamudi et al. 1993, Vadlamudi et al. 1995), and calnexin is a molecular chaperone that participates in potassium channels (Higgins et al. 2003).

Markers With Potentially "Null" Alleles. The second part of Table 3 provides a list of 51 markers that had no amplification in three or more of the samples, or potentially null alleles. In this group, some sequences had significant hits on BLASTx. Among these markers, Stv_Spf688 and Stv_Spf1243 had similarity to a dynactin of $A$. mellifera and to a pyruvate kinase, respectively. Dynactin in $A$. mellifera has shown different degrees of methylation in workers than in queens and is a possible link to environmental response and plasticity (Moczek and Snell-Rood 2008). In marker Stv_Spf688, we observed apparent "null" alleles in all samples from PR. As for pyruvate kinase, this enzyme in Tenebrio molitor $(\mathrm{L}$.) varies in expression depending on the developmental stage of the insect and in response to stress, including application of insecticide (Papadopoulos et al. 2005). In addition, markers Stv Spf74 and Stv Spf1713 had similarity to reverse transcriptases. Also, within this 51-marker group in Table 3, some had significant hits on BLASTn. The most interesting markers were Stv Spf1230, with similarity to a larval serum protein in Bombyx mori (L.) and Stv_Spf1363, with similarity to a Spodoptera litura (F.) nucleopolyhedrovirus (II). Although the markers in this group would not be suitable for direct fingerprinting, it would be interesting to confirm the presence of null alleles and to determine the genetic changes that cause them.

The large number of microsatellite markers for $S$. frugiperda presented in the present work provides a first insight into biological processes that could potentially be affected by genetic variations. Though this work is a preliminary screening of markers, these could become an effective tool for population studies by using multiple loci to help better understand migrations and possible crosses of this insect as well as assist in breeding programs and management practices.

\section{Acknowledgments}

We thank Mary Duke for library pyrosequencing in the Roche 454 and Sheron A. Simpson for testing the SSR markers. This work was supported by USDA-ARS project 640221310-003-00.

\section{References Cited}

Adamczyk, J. J., Jr., J. W. Holloway, B. R. Leonard, and J. B. Graves. 1997. Susceptibility of fall armyworm collected from different plant hosts to selected insecticides and transgenic Bt cotton. J. Cotton Sci. 1: 21-28.

Altschul, S. F., W. Gish, W. Miller, E. W. Myers, and D. J. Lipman. 1990. Basic local alignment search tool. J. Mol. Biol. 215: 403-410.

Arias, R. S., L. E. Ballard, and B. E. Scheffler. 2009. UPIC: Perl scripts to determine the number of SSR markers to run. Bioinformation 3: 353-361.

Blanco, R., M. Portilla, C. A. Abel, H. Winters, R. D. Ford, and D. Streett. 2009. Soybean flower and wheat germ proportions in artificial diet and their effect on the growth rates of the tobacco budworm, Heliothis virescens. J. Insect Sci. 9: 59 (insectscience.org/9.59).

Blanco, C. A., M. Portilla, J. L. Jurat-Fuentes, J. F. Sanchez, D. Viteri, P. Vega-Aquino, A. P. Teran-Vargas, A. AzuaraDominguez, J. D. Lopez, R. S. Arias, et al. 2010. Susceptibility of isofamilies of Spodoptera frugiperda (Lepidoptera: Noctuidae) to CrylAc and CrylFa proteins of Bacillus thuringiensis. Southwest. Entomol. 35: 409-415.

Brownstein, M. J., J. D. Carpten, and J. R. Smith. 1996. Modulation of non-templated nucleotide addition by tag DNA polymerase: primer modifications that facilitate genotyping. Biotechniques 20: 1004-1006, 1008-1010.

Chilcutt, C. F., G. N. Odvody, J. C. Correa, and J. Remmers. 2007. Effects of Bacillus thuringiensis transgenic corn on corn earworm and fall armyworm (Lepidoptera: Noctuidae) densities. J. Econ. Entomol. 100: 327-334.

de Souza, J., S. Jain, C. de Oliveira, C. Ayres, and W. Lucena. 2009. Toxicity of a Bacillus thuringiensis israelensis-like strain against Spodoptera frugiperda. Biocontrol 54: 467473.

Doucet, D., M. G. Tyshenko, P. L. Davies, and V. K. Walker. 2002. A family of expressed antifreeze protein genes from the moth, Choristoneura fumiferana. Eur. J. Biochem. 269: 38-46.

Efron, B., E. Halloran, and S. Holmes. 1996. Bootstrap confidence levels for phylogenetic trees. Proc. Natl. Acad. Sci. U.S.A. 93: 13429-13434.

Ellegren, H. 2004. Microsatellites: simple sequences with complex evolution. Nat. Rev. Genet. 5: 435-445.

Felsenstein, J. 1985. Confidence-limits on phylogenies-an approach using the bootstrap. Evolution 39: 783-791.

Glenn, T. C., and N. A. Schable. 2005. Isolating microsatellite DNA loci. Methods Enzymol. 395: 202-222.

Hashimoto, K., Y. Yamano, and I. Morishima. 2008. Cloning and expression of a gene encoding gallerimycin, a cysteine-rich antifungal peptide, from eri-silkworm, Samia cynthia ricini. Comp. Biochem. Physiol. B Biochem. Mol. Biol. 150: 229-232. 
Hayden, M. J., G. Good, and P. J. Sharp. 2002. Sequence tagged microsatellite profiling (STMP): improved isolation of DNA sequence flanking target SSRs. Nucleic Acids Res. 30: e129.

Hegedus, D. D., M. Erlandson, D. Baldwin, X. W. Hou, and M. Chamankhah. 2008. Differential expansion and evolution of the exon family encoding the Serpin-1 reactive centre loop has resulted in divergent serpin repertoires among the Lepidoptera. Gene 418: 15-21.

Higgins, M. K., M. Demir, and C. G. Tate. 2003. Calnexin coexpression and the use of weaker promoters increase the expression of correctly assembled Shaker potassium channel in insect cells. Biochim. Biophys. Acta Biomembr. 1610: $124-132$.

Hruska, A. J., and S. M. Gladstone. 1988. Effect of period and level of infestation of the fall armyworm Spodoptera frugiperda on irrigated maize yield. Fla. Entomol. 71: 249254.

Ibrahim, K. M., Y. Yosuf, and E. Amna. 2004. Polymerase chain reaction primers for polymorphic microsatellite loci in the African armyworm, Spodoptera exempta (Lepidoptera: Noctuidae). Mol. Ecol. Notes 4: 653-655.

Index-Mundi. 2009. Corn production by country. (http:// www.indexmundi.com/agriculture/? commodity $=$ corn\&graph $=$ production)

Jenkins, J. N. 1999. Transgenic plants expressing toxins from Bacillus thuringiensis, pp. 211-232. In F. R. Hall and J. J. Menn (eds.), Methods in biotechnology. Humana Press, Totowa, NJ.

Johnson, S. J. 1987. Migration and life history strategy of the fall armyworm, Spodoptera frugiperda in the Western Hemisphere. Insect Sci. Appl. 8: 543-549.

Kijas, J. M., J. C. Fowler, C. A. Garbett, and M. R. Thomas. 1994. Enrichment of microsatellites from the citrus genome using biotinylated oligonucleotide sequences bound to streptavidin-coated magnetic particles. Biotechniques 16: 656-660.

Lu, Y. J., G. D. Kochert, D. J. Isenhour, and M. J. Adang. 1994. Molecular characterization of a strain-specific repeated DNA sequence in the fall armyworm Spodoptera frugiperda (Lepidoptera: Noctuidae). Insect Sci. Appl. 3: 123-130.

Lwalaba, D., S. Weidlich, K. H. Hoffmann, and J. Woodring. 2010. Exogenous and endogenous protease inhibitors in the gut of the fall armyworm larvae, Spodoptera frugiperda. Arch. Insect Biochem. Physiol. 74: 114-126.

Machado, V., M. Wunder, V. D. Baldissera, J. V. Oliveira, L. M. Fiuza, and R. N. Nagoshi. 2008. Molecular characterization of host strains of Spodoptera frugiperda (Lepidoptera: Noctuidae) in southern Brazil. Ann. Entomol. Soc. Am. 101: 619-626.

Marenco, R. J., R. E. Foster, and C. A. Sanchez. 1992. Sweet corn response to fall armyworm (Lepidoptera: Noctuidae) damage during vegetative growth. J. Econ. Entomol. 85: 1285-1292.

Martinelli, S., R. M. Barata, M. I. Zucchi, M. C. Silva-Filho, and C. Omoto. 2006. Molecular variability of Spodoptera frugiperda (Lepidoptera: Noctuidae) populations associated to maize and cotton crops in Brazil. J. Econ. Entomol. 99: 519-526.

Moczek, A. P., and E. C. Snell-Rood. 2008. The basis of bee-ing different: the role of gene silencing in plasticity. Evol. Dev. 10: 511-513.

Nagoshi, R. N. 2010. The fall armyworm triose phosphate isomerase (Tpi) gene as a marker of strain identity and interstrain mating. Ann. Entomol. Soc. Am. 103: 283-292.
Nagoshi, R. N., and R. L. Meagher. 2003. FR tandem-repeat sequence in fall armyworm (Lepidoptera: Noctuidae) host strains. Ann. Entomol. Soc. Am. 96: 329-335.

Nagoshi, R. N., and R. L. Meagher. 2008. Review of fall armyworm (Lepidoptera: Noctuidae) genetic complexity and migration. Fla. Entomol. 91: 546-554.

Nagoshi, R. N., and R. L. Meagher. 2009. Behavior and distribution of the two fall armyworm host strains in Florida. Fla. Entomol. 87: 440-449.

Nagoshi, R. N., R. L. Meagher, K. Flanders, J. Gore, R. Jackson, J. Lopez, J. S. Armstrong, G. D. Buntin, C. Sansone, and B. R. Leonard. 2008. Using haplotypes to monitor the migration of fall armyworm (Lepidoptera: Noctuidae) corn-strain populations from Texas and Florida. J. Econ. Entomol. 101: 742-749.

Nagoshi, R. N., F. Fleischer, and R. L. Meagher. 2009. Texas is the overwintering source of fall armyworm in central Pennsylvania: implications for migrati' on into the northeastern United States. Environ. Entomol. 38: 1546-1554.

Nagoshi, R. N., R. L. Meagher, and D. A. Jenkins. 2010. Puerto Rico fall armyworm has only limited interactions with those from Brazil or Texas but could have substantial exchanges with Florida populations. J. Econ. Entomol. 103: 360-367.

Oliveira, E. J., J. G. Pádua, M. I. Zucchi, R. Vencovsky, and M.L.C. Vieira. 2006. Origin, evolution and genome distribution of microsatellites. Genet. Mol. Biol. 29: 294-307.

Papadopoulos, A. I., A. Anagnostis, and D. Lazarou. 2005. Effect of insecticide injection on pyruvate kinase of the insect Tenebrio molitor (Coleopteran). Pestic. Biochem. Physiol. 82: 115-124.

Pashley, D. P. 1986. Host-associated genetic differentiation in fall armyworm (Lepidoptera, Noctuidae) - a sibling species complex. Ann. Entomol. Soc. Am. 79: 898-904.

Pashley, D. P. 1989. Host-associated differentiation in armyworms (Lepidoptera: Noctuidae): an allozymic and mitochondrial DNA perspective, pp. 103-114. In H. D. Loxdale and J. D. Hollander (eds.), Electrophoretic studies on agricultural pests. Systematics Association, Oxford, United Kingdom.

Pashley, D. P., S. J. Johnson, and A. N. Sparks. 1985. Genetic population-structure of migratory moths-the fall armyworm (Lepidoptera, Noctuidae). Ann. Entomol. Soc. Am. 78: $756-762$

Passoa, S. 1991. Color identification of economically important Spodoptera larvae in Honduras (Lepidoptera: Noctuidae). (Online: July 10, 2010; http://digitalcommons. unl.edu $/$ cgi $/$ viewcontent.cgi? article $=1413 \&$ context $=$ insectamundi).

Polanczyk, R. A., R.F.P. da Silva, and L. M. Fiuza. 2000. Effectiveness of Bacillus thuringiensis strain against Spodoptera frugiperda (Lepidoptera: Noctuidae). Braz. J. Microbiol. 31: 165-167.

Potempa, J., E. Korzus, and J. Travis. 1994. The serpin superfamily of proteinase-inhibitors, structure, function, and regulation. J. Biol. Chem. 269: 15957-15960.

Prowell, D. P., M. McMichael, and J. F. Silvain. 2004. Multilocus genetic analysis of host use, introgression, and speciation in host strains of fall armyworm (Lepidoptera: Noctuidae). Ann. Entomol. Soc. Am. 97: 1034-1044.

Richard, G. F., A. Kerrest, and B. Dujon. 2008. Comparative genomics and molecular dynamics of DNA repeats in eukaryotes. Microbiol. Mol. Biol. Rev. 72: 686-727.

Roelofs, W. L., and A. P. Rooney. 2003. Molecular genetics and evolution of pheromone biosynthesis in Lepidoptera. Proc. Natl. Acad. Sci. U.S.A. 100: 9179-9184. 
Rozen, S., and H. Skaletsky. 2000. Bioinformatics methods and protocols in the series methods in molecular biology, pp. 365-386. Humana Press, Totowa, NJ.

Sharopova, N., M. D. McMullen, L. Schultz, S. Schroeder, H. Sanchez-Villeda, J. Gardiner, D. Bergstrom, K. Houchins, S. Melia-Hancock, T. Musket, et al. 2002. Development and mapping of SSR markers for maize. Plant Mol. Biol. 48: 463-481.

Sparks, A. N. 1986. Fall armyworm (Lepidoptera, Noctuidae) - potential for areawide management. Fla. Entomol. 69: 603-614.

Tabashnik, B. E., J.B.J. Van Rensburg, and Y. Carrière. 2009. Field-evolved insect resistance to Bt crops: definition, theory, and data. J. Econ. Entomol. 102: 2011-2025.

Techen, N., R. S. Arias, N. C. Glynn, Z. Pan, I. Khan, and B. E. Scheffler. 2010. Optimized construction of microsatellite-enriched libraries. Mol. Ecol. Res. 10: 508-515.

Vadlamudi, R. K., T. H. Ji, and L. A. Bulla. 1993. A specific binding-protein from Manduca sexta for the insecticidal toxin of Bacillus thuringiensis subsp.. Berliner. J. Biol. Chem. 268: 12334-12340.

Vadlamudi, R. K., E. Weber, I. H. Ji, T. H. Ji, and L. A. Bulla. 1995. Cloning and expression of a receptor for an insecticidal toxin of Bacillus thuringiensis. J. Biol. Chem. 270: $5490-5494$.

Velez-Arango, A. M., R.E.I. Arango, D. M. Villanueva, E. G. Aguilera, and C.I.B. Saldamando. 2008. Indentificacion de biotipos de Spodoptera frugiperda (Lepidoptera: Noc- tuidae) mediante marcadores mitocondriales y nucleares. Rev. Colomb. Entomol. 34: 145-150.

Wang, Y., and J. Hey. 2010. Estimating divergence parameters with small samples from a large number of loci. Genetics 184: 363-379.

Weber, J. L. 1990. Informativeness of human (Dc-Da) N. (Dg-Dt)N polymorphisms. Genomics 7: 524-530.

Weir, B. S. 1990. Genetic data analysis, pp. 87-125. Sinauer, Sunderland, MA.

Westbrook, J. K., and A. N. Sparks. 1986. The role of atmospheric transport in the economic fall armyworm (Lepidoptera: Noctuidae) infestations in the southeastern United States in 1977. Fla. Entomol. 69: 492-502.

Witzgall, P., P. Kirsch, and A. Cork. 2010. Sex pheromones and their impact on pest management. J. Chem. Ecol. 36: $80-100$.

Young,J. R. 1979. Assessing the movement of fall armyworm (Spodoptera frugiperda) using insecticide resistance and wind patterns, pp. 344-351. In R. L. Rabb and G. G. Kennedy (eds.), Movement of highly mobile insects: concepts and methodology in research, North Carolina State University, NC.

Zane, L., L. Bargelloni, and T. Patarnello. 2002. Strategies for microsatellite isolation: a review. Mol. Ecol. 11: 1-16.

Zhao, X., and G. Kochert. 1993. Phylogenetic distribution and genetic mapping of a (GGC) n microsatellite from rice (Oryza sativa L.). Plant Mol. Biol. 21: 607-614.

Received 7 September 2010; accepted 17 January 2011. 